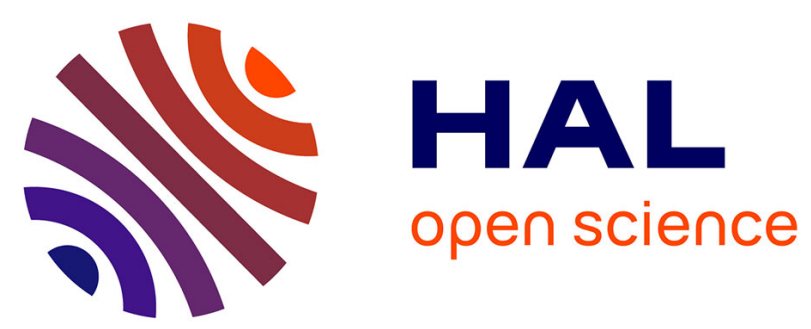

\title{
Homogeneous distribution of fatty ester-based active cosmetic ingredients in hydrophilic thin films by means of nanodispersion
}

\author{
Emilie Munnier, Almar Al Assaad, Stephanie David, Frédéric Mahut, \\ Marylène Vayer, Louise Van Gheluwe, Florent Yvergnaux, Christophe \\ Sinturel, Martin Soucé, Igor Chourpa, et al.
}

\section{To cite this version:}

Emilie Munnier, Almar Al Assaad, Stephanie David, Frédéric Mahut, Marylène Vayer, et al.. Homogeneous distribution of fatty ester-based active cosmetic ingredients in hydrophilic thin films by means of nanodispersion. International Journal of Cosmetic Science, 2020, 42 (5), pp.512-519. 10.1111/ics.12652 . hal-03030342

\section{HAL Id: hal-03030342 https://hal.science/hal-03030342}

Submitted on 30 Nov 2020

HAL is a multi-disciplinary open access archive for the deposit and dissemination of scientific research documents, whether they are published or not. The documents may come from teaching and research institutions in France or abroad, or from public or private research centers.
L'archive ouverte pluridisciplinaire HAL, est destinée au dépôt et à la diffusion de documents scientifiques de niveau recherche, publiés ou non, émanant des établissements d'enseignement et de recherche français ou étrangers, des laboratoires publics ou privés. 
Homogeneous distribution of fatty-esters based active cosmetic ingredients in hydrophilic thin films by means of nanodispersion

Emilie Munnier ${ }^{1, *}$, Almar Al Assaad ${ }^{1}$, Stephanie David ${ }^{1}$, Frederic Mahut ${ }^{2}$, Marylène Vayer ${ }^{2}$, Louise Van Gheluwe ${ }^{1}$, Florent Yvergnaux ${ }^{3}$, Christophe Sinturel ${ }^{2}$, Martin Soucé ${ }^{1}$, Igor Chourpa ${ }^{1}$, Franck Bonnier ${ }^{1}$.

1 Université de Tours, EA 6295 Nanomédicaments et Nanosondes (NMNS), Faculté de Pharmacie, 31 avenue Monge 37200 Tours, France

2 Interfaces, Confinement, Matériaux et Nanostructures (ICMN), CNRS-Université d'Orléans, UMR 7374, 1b, Rue de la Férollerie, C.S. 40059, 45071 Orléans Cedex 2, France

3 Bioeurope (Solabia group), Route d'Oulins, 28260 Anet, France

*Corresponding author emilie.munnier@univ-tours.fr +33 247367201; Fax +33 247367199

Email addresses of all authors:

almar.assaad@hotmail.com

stephanie.david@univ-tours.fr

frederic.mahut@cnrs-orleans.fr

marylene.vayer@univ-orleans.fr

louise.vangheluwe@univ-tours.fr

florent.yvergnaux@solabia.fr

christophe.sinturel@univ-orleans.fr

martin.czok-souce@univ-tours.fr

igor.chourpa@univ-tours.fr

franck.bonnier@univ-tours.fr 


\section{Abstract :}

OBJECTIVE: Cosmetic films and patches are interesting forms to promote skin penetration of active ingredients as they ensure their long stay of the treated zone of the skin. Nevertheless, currently developed films and patches are most of all hydrophilic and are not adapted to the hydrophobic molecules. The aim of this study was to establish if nanodispersion of fatty-acid based active cosmetic ingredients could be a manner to introduce high concentrations of those $\mathrm{ACl}$ in hydrophilic films.

METHODS: Punica granatum seed oil hydroxyphenethyl ester (PHE) is a lipolytic agent obtained by enzymatic conjugation of tyrosol to long chain fatty acids and to enhance its skin diffusion. Nanodispersions of PHE were prepared by a green emulsion-solvent evaporation process and dispersed in polyvinyl alcohol films. Raman imaging coupled to multivariate analysis was used to study the distribution of PHE in the films.

RESULTS: Nanodispersions of PHE combined with antioxidant vitamin E, and stabilized by Pluronic ${ }^{\circledR}$ F127 were successfully prepared. The nanodispersions show a spherical shape and a hydrodynamic diameter close to $100 \mathrm{~nm}$. Raman images analysis with multivariate approaches showed a very homogeneous distribution of PHE nanodispersions in the films compared to free PHE introduced as an ethanolic solution.

CONCLUSION: Nanodispersions of hydrophobic fatty-acid based ingredients seem to be relevant method to introduce this type of ingredient in hydrophilic film matrix. The cosuspension with vitamin $\mathrm{E}$ limits their oxidation in presence of water.

Keywords: nanodispersion, films, Raman imaging, Delivery/vectorization/penetration, Formulation/stability, polymers. 


\section{Introduction}

Fatty acids (FA) are well known in the cosmetic industry as emollients or functional ingredients used to texturize products. Lately, covalently bonding active cosmetic ingredients $(\mathrm{ACl})$ to $\mathrm{FA}$ represents a more and more developed way to enhance skin penetration of small hydrophilic active cosmetic ingredients and increase their activity [1,2]. Moreover, intrinsic properties of some FA, in particular that of polyunsaturated fatty acids (PUFA), confer to cosmetic products antimicrobial [3], antioxidant [4] or anti-aging [5] activity. The low polarity of those esters promotes an easy introduction into the organic phase of emulsion-based products like creams or into products rich in surfactants like shower gels or shampoos. In contrast, introduction of high concentrations of FA into water-based products like gels, lotions or films is limited.

Punica granatum seed oil hydroxyphenethyl ester (PHE) is a commercialized active cosmetic ingredient obtained by enzymatic esterification of pomegranate oil by tyrosol. The resulting active ingredient associates the lipolytic actions of tyrosol and punicic acid, a PUFA. The major and most active molecule of PHE is tyrosyle punicate (Fig. 1).

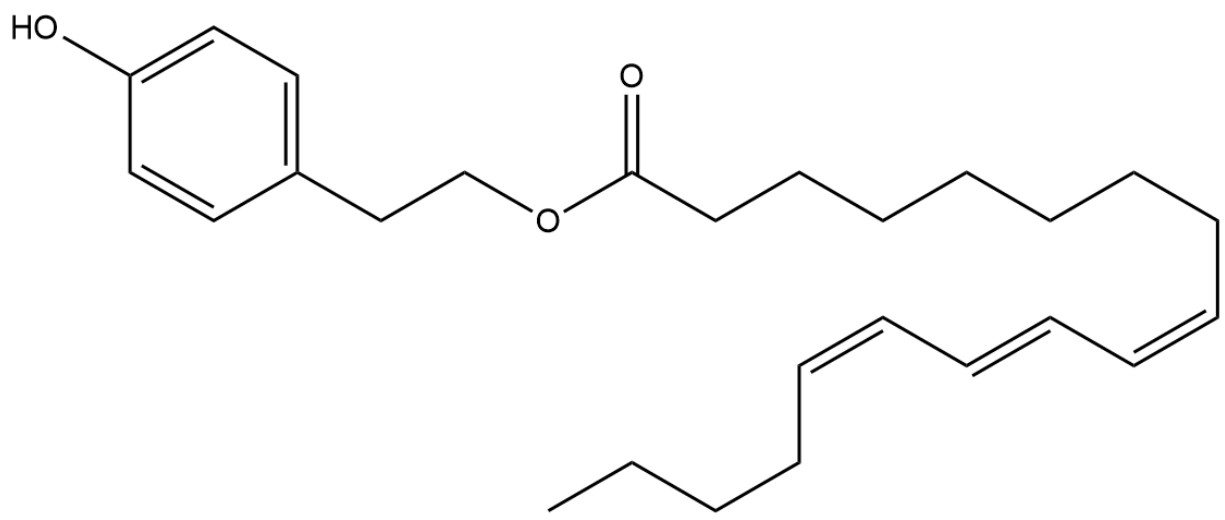

Figure 1: Chemical structure of the major constituent of PHE: tyrosyle punicate

PHE has a melting temperature close to $30^{\circ} \mathrm{C}$ and is semi-solid at room temperature. It is soluble in oils and organic solvents. It has interesting skin penetration properties due to its amphiphilic structure. Nevertheless, it could take advantage of a longer diffusion time at the surface of the skin to increase its concentration in the deep layers, where it exerts its lipolytic action. This $\mathrm{ACl}$ could then benefit of film-forming formulation strategy. Indeed, a film formed in direct contact with the stratum corneum allows topical molecules to have an extended diffusion time [6] and thus favors its penetration in the skin. As film-forming molecules, recent 
cosmetic products integrate predominantly water-soluble polymers, among which polyvinyl alcohol (PVA), polyvinylpyrrolidone, or polysaccharides (alginate, pullulan, chitosan) $[7,8]$. Therefore, it is very difficult to include large amounts of lipid-based active ingredients in these hydrophilic polymers. To address the issue of solubility, current strategy is to add to the preparation some water-miscible organic solvent, most commonly ethanol [9] or isopropanol $[6,10]$. However, the organic solvents do not ensure a homogeneous distribution of the active molecules within the resulting film [11] while they can affect the skin integrity.

It has already been demonstrated that nanoencapsulation of lipophilic molecules can increase their concentration in a gel while keeping its $\mathrm{pH}$, rheological or sensory properties unchanged $[12,13]$. Moreover, nanosystems have also been used to disperse homogeneously an active molecule in films for transdermal or dermal delivery $[6,10,14-17]$. In the present study, a nanodispersion of Punica granatum seed oil hydroxyphenethyl ester (PHE) was prepared by an ultrasound-mediated emulsion evaporation method. A triblock copolymer surfactant, poly(ethylene oxide)-block-poly(propylene oxide)-block-poly(ethylene oxide) (PEO-b-PPO-bPEO) was chosen to stabilize the nanodispersions as this family of polymers proved their ability to stabilize nanosystems and to increase skin penetration of active molecules [18-20]. A natural antioxidant molecule, $\alpha$-tocopherol was co-suspended in the aim of limiting the oxidation of PHE in aqueous environment. The nanodispersions were characterized in terms of size, ACl loading and skin penetration. They were included in a model film of polyvinyl alcohol, usually used as a film forming agent in cosmetic products, like peel off masks or patches. The homogeneity of the distribution of PHE in dry films was explored by Raman imaging coupled to chemometrics.

\section{Materials and methods}

\section{Chemicals}

Punica granatum seed oil hydroxyphenethyl esters (PHE) commercialized as Delipidol ${ }^{\circledR}$ was kindly provided by Solabia (Pantin, France). Pluronic ${ }^{\circledR}$ F127 was kindly provided by BASF (Levallois-Perret, France). Polyvinyl alcohol (Mw: 31000 - 50 000g/mol, 87 - 89\% hydrolyzed) (PVA), $\alpha$-tocopherol, dimethylcarbonate (DMC) were purchased from Sigma Aldrich (SaintQuentin-Fallavier, France). Ultrapure water was obtained with a MilliQ system (Millipore, France). 


\section{Preparation of NanoPHE}

Suspensions of NanoPHE were prepared by the emulsion/evaporation method assisted by ultrasounds [21]. The aqueous phase was prepared by dissolving $600 \mathrm{mg}$ of Pluronic ${ }^{\circledR}$ F127 in 10.5g of ultrapure water. The organic phase was prepared dissolving $1250 \mathrm{mg}$ of PHE and 100 $\mathrm{mg}$ of $\alpha$-tocopherol in $2.75 \mathrm{~g}$ DMC. The nanoemulsion was obtained by sonicating the latter mixture during 2 minutes in a glass rosette cell $(20 \mathrm{kHz}$, amplitude 30\%, Vibracell 75041 Sonics). DMC was then evaporated at $25^{\circ} \mathrm{C}$ (25 mbar $30 \mathrm{~min}$, LABOROTA 40001 - Heidolph) to obtain nanodispersion (NanoPHE). The volume of the preparation was adjusted to $20 \mathrm{~mL}$ with ultrapure water to ensure the reproducibility of the concentration.

\section{Characterization of NanoPHE}

Morphology: Samples were diluted in ultrapure water and deposited on a mica substrate by spin coating before analysis with atomic force microscopy (AFM, Nano-Observer CSInstruments AFM, CSI, France). A FORT type tip (AppNano, $k=2.5 \mathrm{~N} / \mathrm{m} ; \mathrm{f}=60 \mathrm{KhZ}$ ) in a resonant AFM mode was preferred.

Size and surface charge: Average hydrodynamic particle diameter (HD) and polydispersity index (Pdl) of NanoPHE were assessed at $25^{\circ} \mathrm{C}$ using a dynamic light scattering (DLS) instrument (NanoZS, Malvern Instruments, UK). Each sample was diluted 1:10 in ultra-pure water before measurements, with the detection angle at $173^{\circ}$ and a laser wavelength of 633 $\mathrm{nm}$. NanoPHE zeta potential was measured on the same dilution with the same equipment, with the detection angle at $13^{\circ}$ and a $633 \mathrm{~nm}$ red laser. All measurements were carried out in triplicate.

\section{Preparation and characterization of PHE-loaded PVA films}

Films were prepared with the film casting method. PVA/ PHE samples were prepared by mixing a water solution of PVA with a solution of PHE in ethanol (90:10). The mixture was stirred for 30 min. In a similar way, PVA/NanoPHE suspension were prepared by mixing a water solution of PVA with a colloidal suspension of NanoPHE in water. The resulting suspension was stirred for 30 min. For both preparations, the weight ratio between PHE and PVA was 1:10. The films were prepared by scraping the solution between two Scotch ${ }^{\circledR}$ tapes deposited on a glass substrate. Another clean glass slide was used to spread the sample over the entire length of 
the substrate, leaving a liquid film with a constant thickness. The solvent was air-dried under hood for at least $6 \mathrm{~h}$ then the tape is removed leaving the dried film supported on the glass. The films eventually contained $10 \% \mathrm{w} / \mathrm{w}$ PHE.

The thickness of each film was measured on three points to be certain of the reproducibility of the film casting. It was determined using a Micromar 40 EXL digital micrometer (Mahr Technology, France), by subtracting the substrate thickness to the thickness of the area where the film was deposited.

\section{Raman Imaging}

Acquisition: Raman spectral images were acquired using an Alpha 300R microspectrometer (WITec, Ulm, Germany). Spectral data were collected using a $532 \mathrm{~nm}$ DPSS excitation laser source delivering $10 \mathrm{~mW}$ at the sample under a x50 Epiplan HD objective (Zeiss, NA 0.5). The laser power was purposely turned down to avoid photo damaging of samples. Backscattered light was detected by dispersing Raman-shifted radiation onto a Deep Depletion CCD detector (1024x128 pixels) using a grating (600 lines $/ \mathrm{mm}$ ) resulting in a spectral resolution of $\approx 4 \mathrm{~cm}^{-1}$. Raman maps were recorded with $1 \mu \mathrm{m}$ steps in the $\mathrm{x}$ and $\mathrm{y}$ directions and each spectrum resulted from 2 accumulations of 5 seconds. For each condition, three films deposited on three independent slides were analyzed in addition to the reference compounds (PHE, Nano-PHE) and control PVA films containing no PHE.

Data handling: Data analysis was performed using Matlab (MathWorks, USA). Before statistical analysis, Raman spectra were subjected to baseline correction (Lieber correction) followed by vector normalization. Non-negatively Constrained Least Squares analysis (NCLS) is a an unmixing method for spectral data sets initially used to subtract contribution from interferent compound such as paraffin in Raman images collected from skin sections $[22,23]$. Recently, the method has been adapted to study $\mathrm{ACl}$ distribution in permeation studies in reconstructed human skin. Detailed method description can be found in recent work published by Miloudi et al. [24]. The analysis delivers a semi-quantitative estimation (spectral abundance) of the $\mathrm{ACl}$ into the Raman maps. In the present study NCLS is applied to investigate difference in homogeneity of PHE distribution between the two types of polymeric films prepared, namely PVA/PHE and PVA/NanoPHE.

The fitting can be achieved using spectra collected from pure biomolecules. However, it has been demonstrated that distribution of $\mathrm{ACl}$ into a complex matrix can be more accurately 
obtained using reference spectra from blank samples, containing no $\mathrm{ACl}$. Therefore, in the present study Raman images were collected from control PVA films containing no PHE to create a large set of reference spectra accounting for the polymeric matrix. In addition, a pure spectrum of PHE was also integrated during NCLS ultimately to be able to reconstruct abundance map.

\section{Results and discussion}

\section{Preparation of nanodispersion of Punica granatum seed oil hydroxyphenethyl esters}

The research community is now convinced of the interest of nanoformulation in healthcare and well-being [25-27] but is also aware of the urge to adhere to greener ingredients and processes. The use of ultrasound-based methods was recently considered green because it is low-energy consuming [28]. Here, ultrasound-assisted emulsion-evaporation was made even more sustainable by the use of a green solvent, dimethylcarbonate (DMC), instead of traditionally used toxic solvents like methylene chloride [29] to compose the organic phase. To our knowledge, this is the first time that DMC was used to successfully produce nanosystems. Kim et al used DMC to prepare microparticles with mitigated results in terms of reproducibility [30]. The main obstacle evoked by these authors was the relative solubility of DMC in water and the difficulty to evaporate it completely. In our study, the residual DMC in nanodispersions of PHE (NanoPHE) was assessed by gas chromatography. After 30 minutes of rotary evaporation (see Materials and methods part), no DMC was detected (data not shown). The small size, then large surface/volume index of NanoPHE may have permitted an optimal elimination of the solvent during the evaporation phase.

Punicic acid, as every PUFA, can be easily oxidized in presence of water. Despite it was shown that the esterification of PUFAs could slow their oxidation [1], in aqueous nanodispersion, the risk of oxidation is high, because the surface of PHE contact with water is large. In order to reduce the risk of PHE oxidation, $\alpha$-tocopherol (vit E), a widely used lipophilic antioxidant, was added in DMC prior to nanoemulsification for an intimate mix with PHE. Vit E has already proven its ability to protect from oxidation co-encapsulated active molecules [31]. In our study, Vit E concentration was $0.5 \% \mathrm{wt} / \mathrm{wt}$ of the resulting NanoPHE suspension. The optimal formula described in the material and methods section leads to a water suspension of spherical nanosystems with a concentration in PHE of $10 \% \mathrm{wt} / \mathrm{wt}$. The loading in PHE of an 
individual particle is close to $64 \% \mathrm{wt} / \mathrm{wt}$. This high loading could be achieved because no dilution of PHE in a lipid or polymer matrix is necessary during the process developed. Fig. 2 shows individual nanoparticles observed by AFM after deposition on a silicon wafer, exhibiting round particles with a diameter close to $100 \mathrm{~nm}$ and a donut shape (i.e. with a depression in the center of the object). The non-spherical shape observed on the AFM images can be attributed to a sagging of the soft particles on the substrate during drying and/or to deformations induced by the AFM tip. Thus, it is not reflecting the actual shape of the particles in water.

A

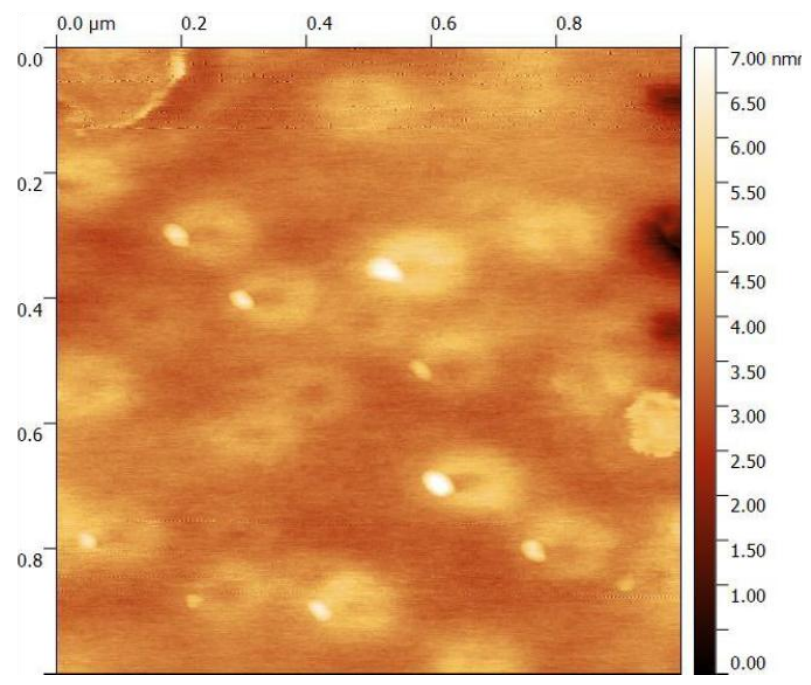

B

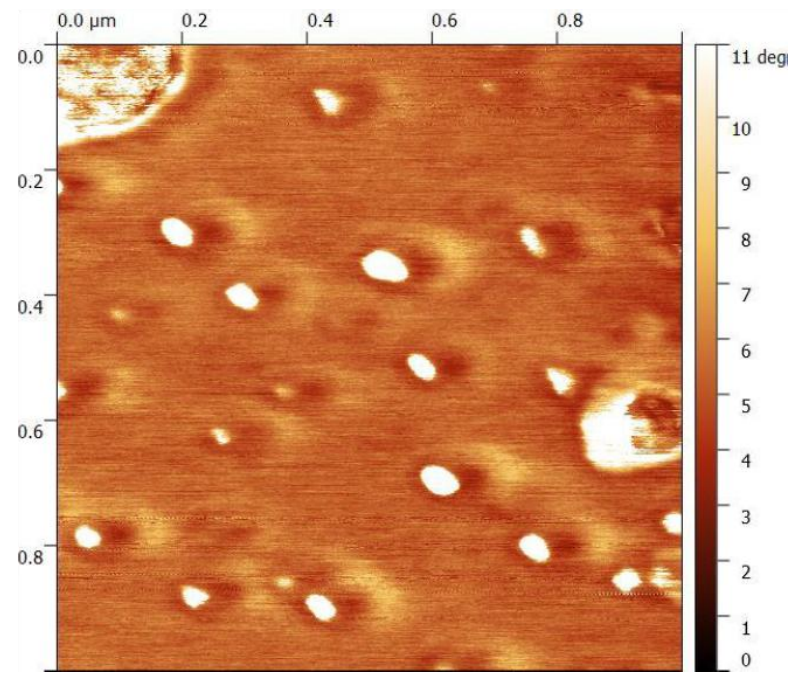

Figure 2: Morphology of NanoPHE as observed by atomic force microscopy A) Topography mode B)

Phase image

NanoPHE samples typically displayed hydrodynamic diameter values of $105 \mathrm{~nm} \pm 5 \mathrm{~nm}$ (Table I). They could be introduced without destabilization in a water-based cosmetic product as their size was not significantly modified by dilution up to 1:1000 (111 $\pm 2 \mathrm{~nm})$. Nanodispersions of punicic acid monoglycerides, a close compound, were previously prepared by Cao et al for nutrition application [32]. A similar process involving ultrasounds was employed producing nanoobjects with an average size around $6 \mathrm{~nm}$. In the present study, objects with a $D_{H}$ of ca $100 \mathrm{~nm}$ are more compatible with cosmetic applications.

For the initial NanoPhe, the polydispersity index was inferior to 0.15 , showing samples were nearly monodisperse. After 1:1000 dilution, the suspensions were monodisperse, with the Pdl values of 0.077 . The zeta potential is slightly negative and reproducible $(\approx-11 \mathrm{mV})$. 
Electrostatic repulsion of the particles was not an issue as the colloidal stability was expected to be ensured by the steric hindrance of the PEO chains of Pluronic ${ }^{\circledR} F 127$ stabilizing the system [33]. Colloidal characteristics were stable after a shelf life of three months at room temperature (Table I).

Table I: Physicochemical characteristics of NanoPHE, initially or after one month of shelf life at room temperature

\begin{tabular}{cccc}
\hline & $\begin{array}{c}\text { Hydrodynamic } \\
\text { diameter }(\mathrm{nm})\end{array}$ & Polydispersity index & $\begin{array}{c}\text { Zeta potential } \\
(\mathrm{mV})\end{array}$ \\
\hline NanoPHE & $105 \pm 5$ & $0.114 \pm 0.010$ & $-11.9 \pm 2.5$ \\
$\begin{array}{c}\text { NanoPHE, } \\
3 \text { months of storage }\end{array}$ & $111 \pm 2$ & $0.068 \pm 0.017$ & $-11.3 \pm 2.8$ \\
\hline
\end{tabular}

\section{Dispersion of PHE in polyvinyl alcohol films}

Films were prepared on a glass slide by air-drying a solution containing the film-forming agent PVA and commercial PHE dissolved in ethanol $(10 \% \mathrm{~V} / \mathrm{V})$ or NanoPHE. There is naturally a large variability in thickness of films applied onto the skin by customers depending on their usage of products and amounts applied. Commonly it is however well spread across large skin surface resulting in thin films of few microns. Even if there is no consensus on the thickness of the residual film after air-drying of a cosmetic product on the skin, for the purpose of the study, 10 $\mu \mathrm{m}$ films were prepared on glass slides enabling reproducible samples preparation and analysis with Raman spectroscopy. The average thickness of the analyzed areas was $9.6 \pm 2.4 \mu \mathrm{m}$.

Raman imaging was performed on control films (containing no PHE), PHE-loaded films with no vitamin E supplementation and NanoPHE-loaded films to study the distribution of ingredients based on their spectral signatures. Each pixel of spectral images collected contained a combination of spectral features originating from the different ingredients. PHE was characterized by a specific Raman signature with main peaks located at $1635 \mathrm{~cm}^{-1}(\mathrm{v} \mathrm{C}=\mathrm{C})$, $1253 \mathrm{~cm}^{-1}(\delta=\mathrm{CH})$ and $1157 \mathrm{~cm}^{-1}$ (v C-C) (Fig. 3, spectrum A). In comparison, PVA spectrum 
(spectrum $D$ in Fig. 3) did not display strong, sharp features, but only few broader bands corresponding to $\mathrm{C}-\mathrm{C}$ stretching at $856 \mathrm{~cm}^{-1}$ and $920 \mathrm{~cm}^{-1}, \mathrm{C}-\mathrm{H}$ and $\mathrm{O}-\mathrm{H}$ bending at $1360 \mathrm{~cm}^{-1}$ and $1440 \mathrm{~cm}^{-1}$. Additional bands of PVA, i.e. C-O stretching, $\mathrm{O}-\mathrm{H}$ banding and $\mathrm{C}-\mathrm{C}$ stretching can be found between $1000-1150 \mathrm{~cm}^{-1}$ without clear distinction due to overlapping of peaks [34]. As a result, PHE and PVA exhibit strong contrast in band positions and intensities with reduced overlapping between features. Although NanoPHE are chemically complex structures containing Pluronic ${ }^{\circledR}$ F127 at the surface and a mixture of PHE/ $\alpha$-tocopherol into the core, the Raman spectrum of NanoPHE (spectrum B in Fig. 3) remained similar to that of pure PHE. This is explained by the particularly intense Raman response of PHE compared to Pluronic ${ }^{\circledR}$ F127 and enhanced by the low concentration $\alpha$-tocopherol in nanodispersions. The molecular fingerprint delivered by Raman spectroscopy enables to witness spectral modifications in chemical structures. For instance, PHE can be subject to degradation by oxidation, which is characterized by specific spectral features (Fig. 3, spectrum C). The strong decrease in the main feature intensity at $1630 \mathrm{~cm}^{-1}$ in the degraded form of PHE lead to other weaker bands to appear more intense due to changes in band ratio. Despite change in scaling, it was observed that there was no difference in features at $644 \mathrm{~cm}^{-1}, 824 \mathrm{~cm}^{-1}, 842 \mathrm{~cm}^{-1}, 1444 \mathrm{~cm}^{-1}$ between the native PHE and the oxidized PHE (Fig. 3, spectra A and C, respectively). In contrast, the most obvious modifications in the Raman spectrum of degraded NanoPHE were the presence of a double peak at $1635 \mathrm{~cm}^{-1}-1660 \mathrm{~cm}^{-1}$, but also a shift in the band at $1157 \mathrm{~cm}^{-1}$ to $1174 \mathrm{~cm}^{-}$ ${ }^{1}$ and a shoulder in the band at $1244 \mathrm{~cm}^{-1}$. 


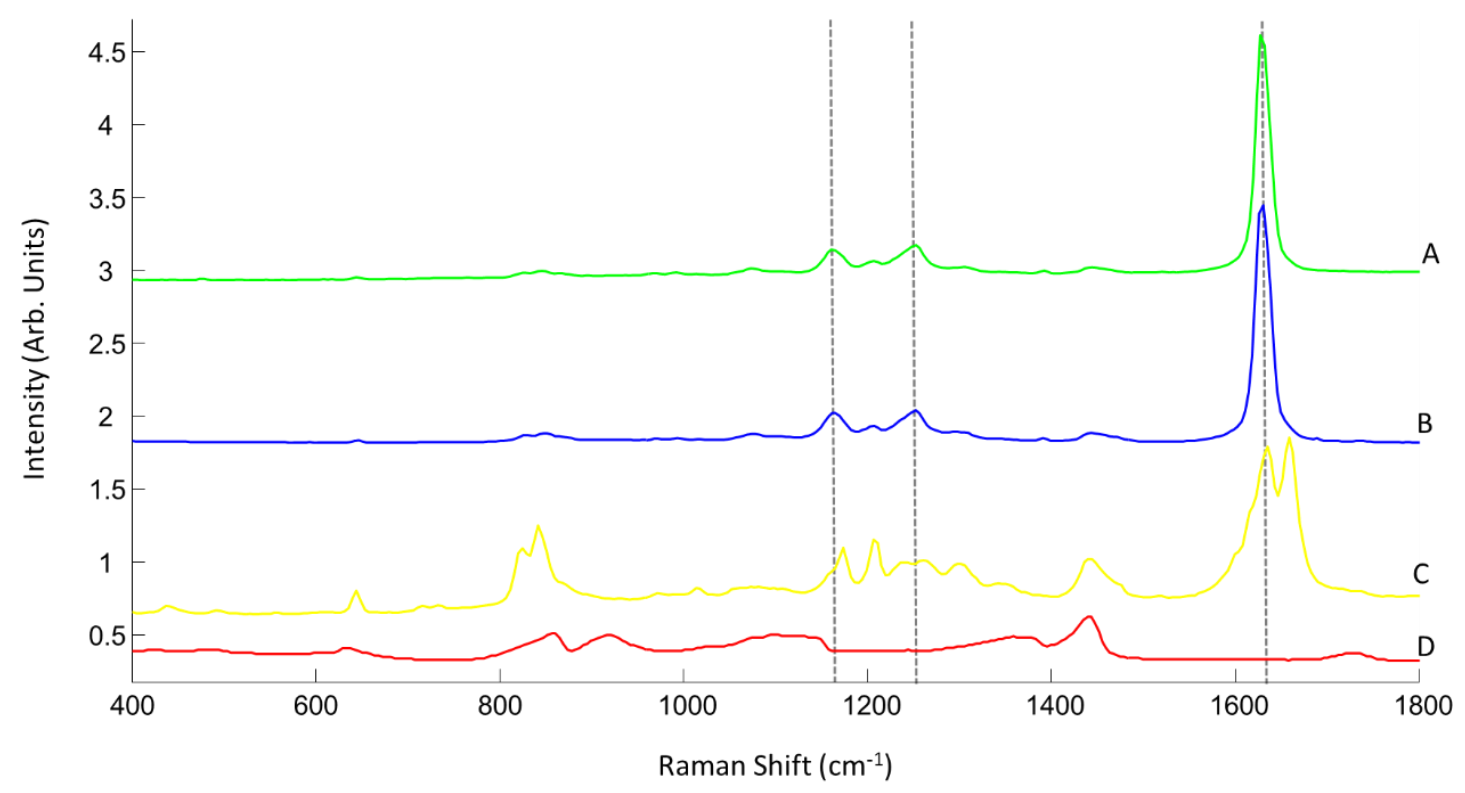

Figure 3 Raman Spectra of main compounds: (A) PHE, (B) NanoPHE, (C) degraded PHE and (D) PVA control. Spectra offset for clarity. Dotted line highlights most relevant features of PHE.

Fig. 4 shows bright field images for PVA films with free PHE and NanoPHE. While PVA films with free PHE display a number of spherical objects unevenly distributed across the field of view, PVA films with NanoPHE appear visually uniform. Raman bands intensity maps at 1440 $\mathrm{cm}^{-1}$ (main PVA feature), $1635 \mathrm{~cm}^{-1}$ (main PHE feature) and $1654 \mathrm{~cm}^{-1}$ (found in degraded PHE) enables to observe different patterns in ingredients distribution. For PVA-PHE film, spectra exhibit different intensities in the PHE feature at $1635 \mathrm{~cm}^{-1}$ across the analyzed area suggesting the $\mathrm{ACl}$ is unevenly distributed (Fig. 4B, spectra $\mathrm{a}$ and b). The comparison of intensity maps at $1635 \mathrm{~cm}^{-1}$ and $1654 \mathrm{~cm}^{-1}$ add further emphasis on the presence of areas with high accumulation of degraded PHE while other display only native PHE (Fig. 4B, spectrum c). Distribution of PHE in PVA-NanoPHE appears more homogenous and spectrum d in Fig. 4B presents the typical spectral signature observed across the scanned area. Images constructed from single bands provide an overview of the data but should be interpreted with caution. Raman microspectroscopy is sensitive to focal volume position on the surface of samples during recording that can lead to substantial changes in intensity between pixels possibly leading to false assumption. For instance intensity maps at $1440 \mathrm{~cm}^{-1}, 1635 \mathrm{~cm}^{-1}$ and $1654 \mathrm{~cm}^{-1}$ in Fig. 4A exhibit dark blue spherical-like structures that could be incorrectly interpreted as absence of PVA and PHE, while they correspond to areas delivering less intense Raman spectra (data not shown). This could be simply explained by low samples density (air bubbles) or 
irregularity on the surface causing less efficient focusing thus an overall drop of intensity in those pixels.

A

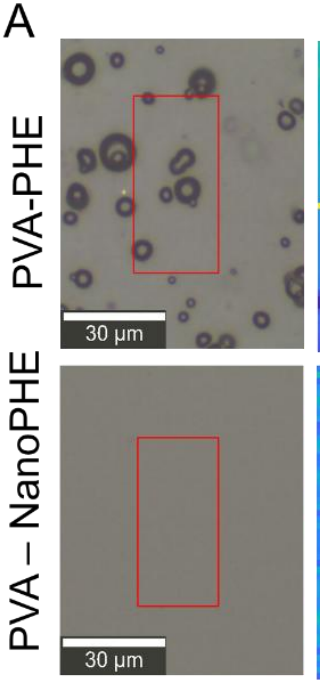

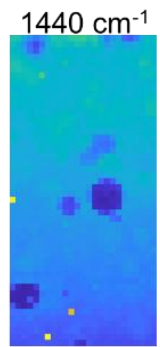

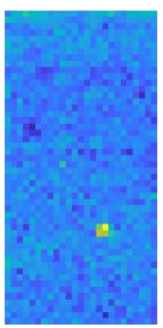

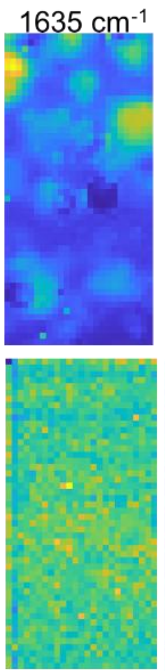

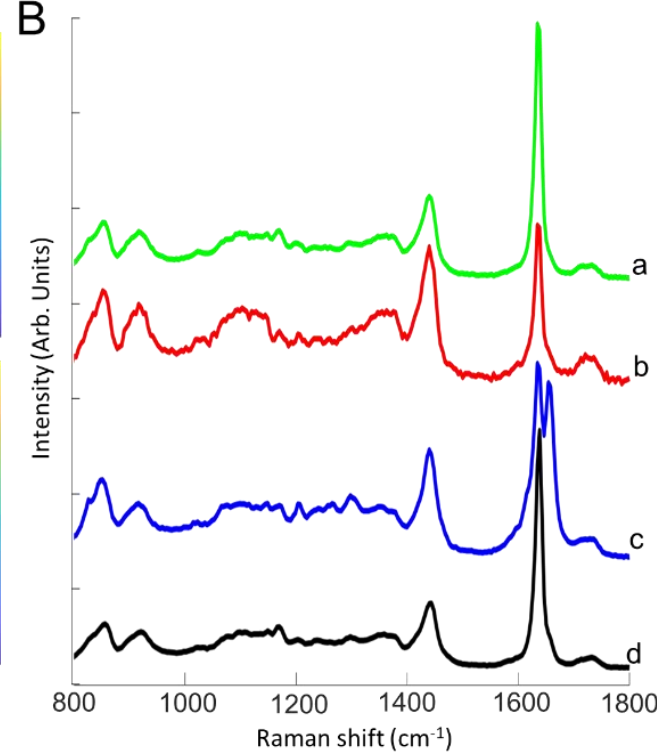

Figure 4: A: White light images of the PVA PHE and PVA-NAnOPHE films with corresponding intensity maps at $1440 \mathrm{~cm}^{-1}, 1635 \mathrm{~cm}^{-1}$ and $1654 \mathrm{~cm}^{-1}$. B: Raman spectra extracted from position labelled $a, b, c$ and $d$ on images. Spectra offset for clarity.

In order to refine the data analysis and overcome such artifacts, NCLS coupled to preprocessing of Raman images was performed. It is a multivariate approach taking into account the whole spectrum rather than a single wavenumber. It is considered that Raman spectra in each pixel of the image result from the combined contribution of all constituents. Therefore, using a set of pure reference spectra, the algorithm estimates the contribution (or abundance) of each compound to the signal. It computes a simulated spectrum from reference spectra provided adjusting their abundances until a result as close as possible to the experimental spectrum collected from the sample is reached. Presently, control films containing only PVA were used to generate reference spectra of polymers while spectra collected from both forms of PHE (native and oxidized) were included in the model to estimate the overall PHE abundance. Ultimately, a semi-quantitative reconstructed false color maps is obtained displaying the distribution of a given compound.

Fig. 5 shows reconstructed chemical maps displaying total PHE contribution in three different PVA-PHE (Fig. 5a, b and c) and Nano-PHE films (Fig. 5d, e and f). All reconstructed images are 
shown on the same scale between 0 and 1 , with 1 equal to $100 \%$, corresponding to pixels displaying a spectrum identical to one pure reference spectrum.

(a)

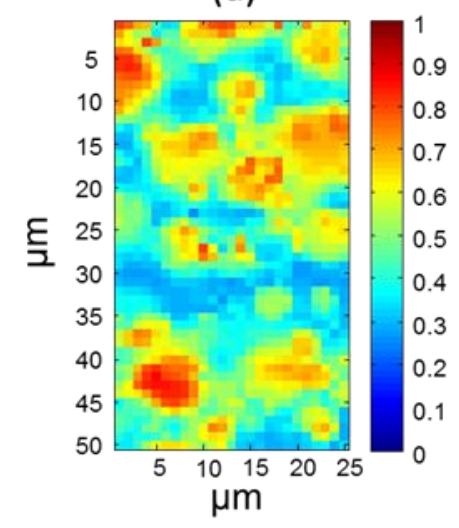

(d)

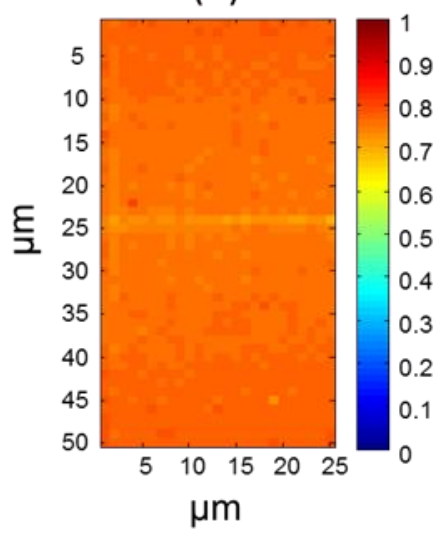

(b)

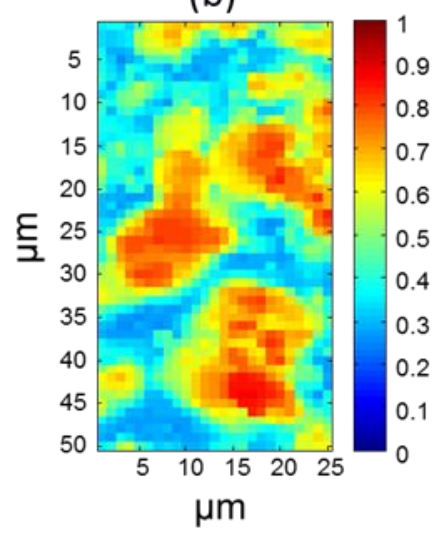

(e)

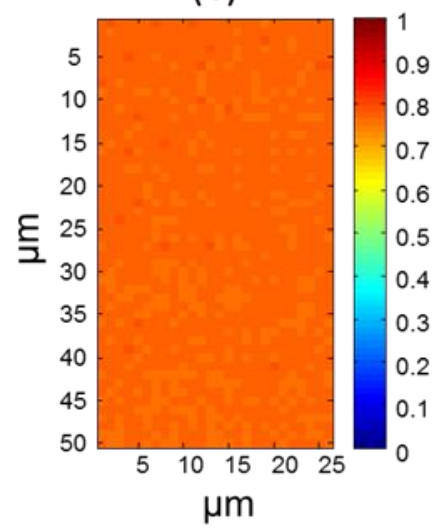

(c)

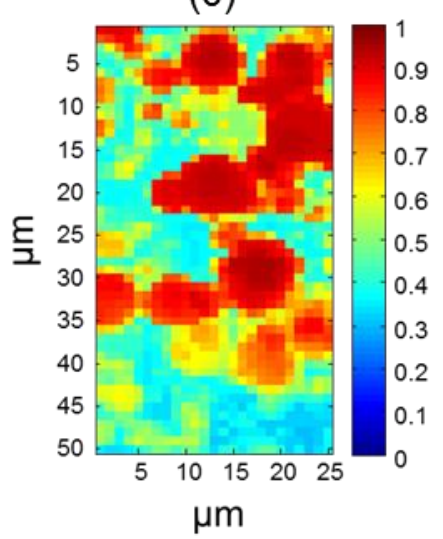

(f)

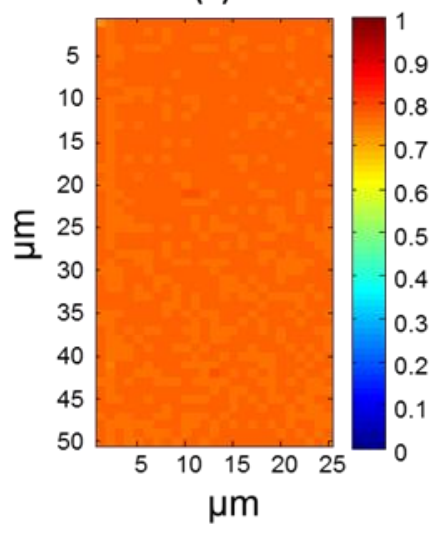

Figure 5: Reconstructed non negatively constrained least squares (NCLS) maps showing the distribution of PHE in PVA films. PVA-PHE films (a), (b) and (c) compared to PVA - NanoPHE films (d), (e) and (f). Maps for each condition are provided from 3 different films. PHE is present at the same concentration in all the samples and the color scale bar indicates PHE relative spectral abundance.

Free PHE integrated to PVA films via ethanol solution generated highly heterogeneous distributions with, on the same map, areas accounting for 10-20\% of PHE beside concentrated droplets where values over $80 \%$ of PHE was reached. Once PHE was nanodispersed, the intensity maps appeared more constant across spectral images suggesting a higher degree of homogeneity in the PHE distribution. . The algorithm is considering multiple bands positions and ratio during calculations of compounds abundance in each pixel consequently artefacts (blue spherical structures) observed previously in single wavenumbers intensity are not 
witnessed after NCLS. The approach is a rapid tool to appreciate PHE distribution while minimizing experimental and instrumental interferences naturally present in Raman data. The mean values of the intensities with respective standard deviation were calculated to be $0.533 \pm 0.149$ (Fig. 5a), $0.558 \pm 0.177$ (Fig. 5b) and $0.646 \pm 0.203$ (Fig. 5c) compared to $0.736 \pm 0.01$ (Fig. 5d), $0.734 \pm 0.01$ (Fig. 5e) and 0.724+0.005 (Fig. 5f). The spectral signature collected differ greatly depending on the type of chemical bonds found in samples analyzed. The $V C=C$ is particularly active vibrational mode in Raman spectra leading to a sharp and intense peak at $1635 \mathrm{~cm}^{-1}$ while in the oxidized form the features in this spectral range are significantly weaker. Raman spectroscopy coupled to NCLS remains a semi-quantitative technique delivering spectral abundance of given compounds. Presently, the results reflect the contribution of PHE compared to PVA, therefore the reduced intensities of main features its oxidized form would also decrease its relative abundance. That explains the difference found in mean values calculated from NCLS. Moreover, Raman imaging was not proposed to determine absolute PHE concentrations but to study distribution into films. The means however highlight specific trends between both types of films analyzed with triplicates performed on three different films delivering similar outcomes. In order to better interpret the information of reconstructed false color maps the standard deviations is more relevant. Globally standard deviations from NanoPHE films are significantly lower compared to PVA-PHE films. For NanoPHE films standard deviation of $0.149,0.177$ and 0.203 respectively represent values of standard deviation represent $27.9 \%, 31.7 \%$ and $31.4 \%$ of means values while for PVAPHE films $0.01,0.01$ and 0.005 respectively represent $1.4 \%, 1.4 \%$ and $0.7 \%$.

Commonly cosmetic or therapeutic films are characterized in terms of morphology, mechanical properties, qualitative chemical composition or total contents after destruction $[6,8]$. Here is proposed an innovative non-destructive approach to evaluate the homogeneity of distribution of molecules of interest in thin films. Moreover, Raman spectroscopy combined to multivariate analysis enables to generate revealing graphical representations and estimated numerical values reflecting homogeneity and integrity of films analyzed. This approach could be a very efficient tool to validate in a short time a cosmetic product formula by attesting of its homogeneity in $\mathrm{ACl}$. It could be also used to study films quality on substrates more realistic than glass slides, like for example polymer-based skin models [35] or reconstructed skin models. Presently, this approach highlights two significant interests of NanoPHE. First, the distribution of PHE is more homogenous in the film when introduced as a nanodispersion than as an organic solution. Secondly, the analysis demonstrated that the co-dispersion of VitE 
permitted to protect PHE from oxidation during the steps of preparation of the nanodispersion, film formulation and ultimately into dried films. 


\section{Conclusion}

In this preliminary study, nanodispersions of PUFA-esters combined with antioxidant vitamin E and stabilized with Pluronic ${ }^{\circledR}$ F127 where successfully prepared by a sustainable method. Their hydrodynamic diameter close to $100 \mathrm{~nm}$ is suitable for an introduction in a cosmetic or pharmaceutical product intended for skin care. The resulting aqueous suspensions showed a long term colloidal stability (over three months) and were used to prepare homogeneous PHEloaded hydrophilic films without the use of organic solvents. The analysis of nanocomposite films by Raman spectroscopy coupled to chemometrics demonstrated that NanoPHE enriched in Vit E protected the PHE from oxidation in air-dried films. The results presented here are promising for a perspective of applying the nanodispersion and film strategy not only to PHE but also to other long-chain PUFA-derived $\mathrm{ACl}$.

\section{Acknowledgments}

We want to thank CSI for the technical assistance for the AFM measurements. We thank Région Centre-Val de Loire for the funding (MISTIC project ARD 2020 Cosmétosciences 201700118114 and Fonds Européen de Développement Régional).

\section{References}

[1] Couturier L and Yvergnaux F 2009 Combined structural and biological activities for new polyunsaturated fatty derivatives obtained by biotechnological process Int. J. Cosmet. Sci. 31 209-24

[2] Sun Y, Zhou D and Shahidi F 2018 Antioxidant properties of tyrosol and hydroxytyrosol saturated fatty acid esters Food Chem. 245 1262-8

[3] Boutin R, Munnier E, Renaudeau N, Girardot M, Pinault M, Chevalier S, Chourpa I, Clément-Larosière B, Imbert C and Boudesocque-Delaye L 2019 Spirulina platensis sustainable lipid extracts in alginate-based nanocarriers: An algal approach against biofilms Algal Res.

[4] Richard D, Kefi K, Barbe U, Bausero P and Visioli F 2008 Polyunsaturated fatty acids as antioxidants Pharmacol. Res. $\mathbf{5 7}$ 451-5

[5] Liu C, Hu J, Sui H, Zhao Q, Zhang X and Wang W 2017 Enhanced skin permeation of glabridin using eutectic mixture-based nanoemulsion Drug Deliv. Transl. Res. 7 325-32 
[6] Tran T T D and Tran P H L 2019 Controlled Release Film Forming Systems in Drug Delivery : The Potential for E ffi cient Drug Delivery 1-16

[7] Kathe K and Kathpalia $\mathrm{H} 2017$ Film forming systems for topical and transdermal drug delivery Asian J. Pharm. Sci. 12 487-97

[8] Karki S, Kim H, Na S J, Shin D, Jo K and Lee J 2016 Thin films as an emerging platform for drug delivery Asian J. Pharm. Sci. 11 559-74

[9] Al-Nemrawi N K and Dave R H 2016 Formulation and characterization of acetaminophen nanoparticles in orally disintegrating films Drug Deliv. 23 540-9

[10] Zaid Alkilani A, Hamed R, Al-Marabeh S, Kamal A, Abu-Huwaij R and Hamad I 2018 Nanoemulsion-based film formulation for transdermal delivery of carvedilol J. Drug Deliv. Sci. Technol. 46 122-8

[11] Dammak I and Sobral P J do A 2019 Active gelatin films incorporated with eugenol nanoemulsions: effect of emulsifier type on films properties Int. J. Food Sci. Technol. 111

[12] Nguyen H T, Munnier E, Souce M, Perse X, David S, Bonnier F, Vial F, Yvergnaux F, Perrier T, Cohen-Jonathan S and Chourpa I 2015 Novel alginate-based nanocarriers as a strategy to include high concentrations of hydrophobic compounds in hydrogels for topical application Nanotechnology 26

[13] Nguyen H T P, Soucé M, Perse X, Vial F, Perrier T, Yvergnaux F, Chourpa I and Munnier E 2017 Lipid-based submicron capsules as a strategy to include high concentrations of a hydrophobic lightening agent in a hydrogel Int. J. Cosmet. Sci. 39

[14] Contri R V., Kulkamp-Guerreiro I C, da Silva S J, Frank L A, Pohlmann A R and Guterres S S 2016 Nanoencapsulation of Rose-Hip Oil Prevents Oil Oxidation and Allows Obtainment of Gel and Film Topical Formulations AAPS PharmSciTech 17 863-71

[15] Brum A A S, Santos P P dos, Silva M M da, Paese K, Guterres S S, Costa T M H, Pohlmann A R, Jablonski A, Flôres S H and Rios A de O 2017 Lutein-loaded lipid-core nanocapsules: Physicochemical characterization and stability evaluation Colloids Surfaces A Physicochem. Eng. Asp. 522 477-84

[16] Niamlang P, Tongrain T, Ekabutr P, Chuysinuan P and Supaphol P 2017 Preparation, 
characterization and biocompatibility of poly(vinyl alcohol) films containing tetracycline hydrochloride-loaded quaternized chitosan nanoparticles J. Drug Deliv. Sci. Technol. 38 $36-44$

[17] Lei K, Wang X, Li X and Wang L 2019 The innovative fabrication and applications of carvacrol nanoemulsions, carboxymethyl chitosan microgels and their composite films Colloids Surfaces B Biointerfaces 175 688-96

[18] Tavano L, Muzzalupo R, Trombino S, Cassano R, Pingitore A and Picci N 2010 Effect of formulations variables on the in vitro percutaneous permeation of Sodium Diclofenac from new vesicular systems obtained from Pluronic triblock copolymers Colloids Surfaces B Biointerfaces 79 227-34

[19] Kahraman E, Özhan G, Özsoy Y and Güngör S 2016 Polymeric micellar nanocarriers of benzoyl peroxide as potential follicular targeting approach for acne treatment Colloids Surfaces B Biointerfaces 146 692-9

[20] Raval A, Pillai S A, Bahadur A and Bahadur P 2017 Systematic characterization of Pluronic ${ }^{\circledR}$ micelles and their application for solubilization and in vitro release of some hydrophobic anticancer drugs J. Mol. Liq. 230 473-81

[21] Tewes F, Munnier E, Antoon B, Ngaboni Okassa L, Cohen-Jonathan S, Marchais H, Douziech-Eyrolles L, Soucé M, Dubois P and Chourpa I 2007 Comparative study of doxorubicin-loaded poly(lactide-co-glycolide) nanoparticles prepared by single and double emulsion methods Eur. J. Pharm. Biopharm. 66

[22] Gobinet C, Sebiskveradze D, Vrabie V, Tfayli A, Piot O and Manfait M 2008 Digital dewaxing of Raman spectral images of paraffin-embedded human skin biopsies based on ICA and NCLS Eur. Signal Process. Conf.

[23] Tfayli A, Gobinet C, Vrabie V, Huez R, Manfait M and Piot O 2009 Digital Dewaxing of Raman Signals: Discrimination between Nevi and Melanoma Spectra Obtained from Paraffin-Embedded Skin Biopsies Appl. Spectrosc. 63 564-70

[24] Miloudi L, Bonnier F, Tfayli A, Yvergnaux F, Byrne H J, Chourpa I and Munnier E 2017 Confocal Raman spectroscopic imaging for in vitro monitoring of active ingredient penetration and distribution in reconstructed human epidermis model J. Biophotonics

[25] Roberts M, Mohammed Y, Pastore M, Namjoshi S, Yousef S, Alinaghi A, Haridass I, Abd 
E, Leite-Silva V, Benson H and Grice J 2017 Topical and cutaneous delivery using nanosystems J. Control. Release 247 86-105

[26] Mihranyan A, Ferraz N and Strømme M 2012 Current status and future prospects of nanotechnology in cosmetics Prog. Mater. Sci. 57 875-910

[27] Goyal R, Macri L K, Kaplan H M and Kohn J 2016 Nanoparticles and nanofibers for topical drug delivery J. Control. Release

[28] Lupacchini M, Mascitti A, Giachi G, Tonucci L, d'Alessandro N, Martinez J and Colacino E 2017 Sonochemistry in non-conventional, green solvents or solvent-free reactions Tetrahedron 73 609-53

[29] Pyo S H, Park J H, Chang T S and Hatti-Kaul R 2017 Dimethyl carbonate as a green chemical Curr. Opin. Green Sustain. Chem. 5 61-6

[30] Kim H, Kim S and Sah H 2018 Solvent hydrolysis rate determines critical quality attributes of PLGA microspheres prepared using non-volatile green solvent J. Biomater. Sci. Polym. Ed. 29 35-56

[31] Zhang F, Khan M A, Cheng H and Liang L 2019 Co-encapsulation of $\alpha$-tocopherol and resveratrol within zein nanoparticles: Impact on antioxidant activity and stability J. Food Eng. 247 9-18

[32] Cao Y, Wang L, He M, Zhang Y and Wang H 2014 Nanodispersions of monoglycerides of punicic acid: A potential nutrient precursor with higher oxidative stability and cytotoxicity RSC Adv. 4 43392-8

[33] Chiper M, Hervé Aubert K, Augé A, Fouquenet J F, Soucé M and Chourpa I 2013 Colloidal stability and thermo-responsive properties of iron oxide nanoparticles coated with polymers: Advantages of Pluronic ${ }^{\circledR}$ F68-PEG mixture Nanotechnology 24

[34] Yang C C, Chiu S J, Lee K T, Chien W C, Lin C T and Huang C A 2008 Study of poly(vinyl alcohol)/titanium oxide composite polymer membranes and their application on alkaline direct alcohol fuel cell J. Power Sources 184 44-51

[35] Gore E, Picard C and Savary G 2018 Biotribology Spreading behavior of cosmetic emulsions : Impact of the oil phase Biotribology 16 17-24 